\title{
10
}

\section{The Role of the Gut in the Neurobiology of Functional Somatic Symptoms}

Abstract Functional somatic symptoms involving the gut are common in children and adolescents. The human gut has a close symbiotic relationship with the microorganisms that live inside it. When this relationship is going well, the microbiota-the community of organisms that live inside the gut — and the gut work together to break down food to provide both the microbiota and the body with energy, and to protect the integrity of the gut barrier (intestinal wall). Working together, the microbiota and gut also modulate set-points within the stress system and contribute to brain function and subjective experience. Because of these wide-ranging functions and because the gut talks to the brain and the brain talks to the gut, functional gut disorders are conceptualized as disorders arising from dysregulation of the microbiota-gut-brain axis. This axis overlaps, interconnects, and interacts with multiple components of the stress system. Activation or dysregulation of the stress system affects gut function and dysregulates the microbiota-gut-brain axis, which then affects stress-system function. In this chapter we examine the interactions between the stress system and microbiota-gut-brain axis. We also discuss the functional somatic symptoms that may arise when that axis is dysregulated. 
Functional somatic symptoms involving the gut-abdominal pain, nausea, vomiting, irregular bowel function, and rumination-are common in children (including adolescents) and are a leading cause of functional impairment and school absenteeism (Varni et al. 2006). Functional gut symptoms sit on a continuum with other functional somatic symptoms and, like other functional somatic symptoms, show an association with adverse childhood experiences (ACEs) and with stress more generally (Bradford et al. 2012; Park et al. 2016; Michels et al. 2019) (for more references see Online Supplement 1.3). These gut symptoms can present as the primary presenting symptom, or they can present alongside other functional somatic symptoms (see, e.g., case of Paula in Chapter 2 or case of Jai in Chapter 5).

Consensus guidelines (the Rome IV Criteria) used by gastroenterologists cluster functional gut symptoms into 10 diagnostic entities in childhood and 33 diagnostic entities in adulthood (Hyams et al. 2016; Drossman and Hasler 2016) (see Text Box 10.1).

\section{Text Box 10.1: Functional gut diagnoses}

When the child with functional gut symptoms goes to see the paediatrician, she may receive a diagnosis that fits under one of three clusters (see Hyams et al. [2016]):

\section{Cluster 1: Functional nausea and vomiting disorders}

- Cyclic vomiting syndrome

- Functional nausea and functional vomiting

- Rumination syndrome (belch-like motor pattern)

- Aerophagia

\section{Cluster 2: Functional abdominal pain disorders}

- Functional dyspepsia

- Irritable bowel syndrome

- Abdominal migraine

- Functional abdominal pain not otherwise specified

Cluster 3: Functional defecation disorders

- Functional constipation

- Nonretentive faecal incontinence 


\section{Shifting Gear to Systems Thinking: A Few Tips to the Reader}

As recently as two decades ago, the gut was considered an independent organ, and functional gut disorders were conceptualized as problems that related to the gut. Over the last decade, however, it has become clear that the gut is part of a complex system-what is known as the microbiota-gut-brain axis. This system includes the microbiota (the organisms living in the gut), the gut itself, the communication pathways between the gut and the brain, and relevant regions in the brain (including the brain stress systems). In turn, functional gut disorders have come to be conceptualized as complex disorders arising from dysregulation of the microbiota-gut-brain axis. This conceptualization reflects the introduction of systems thinking into gastroenterology. Gut doctors are now well aware that the gut does not function as an independent organ but that it must be understood as part of a complex system.

The idea of the microbiota-gut-brain axis brings together many processes on multiple system levels, from the gut lumen (i.e., the inside of the intestine) to the brain (Drossman 2016), including the following:

- The composition and health of the gut microbiota, the colony of organisms that live in the gut lumen.

- The health of the gut itself, including the following: the integrity of the mucosal barrier and immune function; gut motility; digestive processes; and gut signalling.

- The communication from the gut (and the microbiota) to the brain. Key communication pathways include the following: the autonomic nervous system (the vagal nerve carries information from the gut to the brain; see Chapter 6); immune-inflammatory signalling molecules, which are part of the immune-inflammatory system (see Chapter 9); and metabolites and neurotransmitters that are synthesized and processed by the gut (and microbiota). In this way the gut modulates brain function - and function of the HPA axis-bottom-up.

- The communication from the brain to the gut and top-down modulation of gut function (the autonomic nervous system modulates gut function top-down, see Chapter 6). 
- Activation of the pain system on the gut, spinal cord, and brain system levels (see below and Chapter 9).

To add to the complexity, the microbiota-gut-brain axis overlaps, interconnects, and interacts with the stress system-the autonomic nervous system, HPA axis, immune-inflammatory system, and brain stress systems - in many ways. Activation of the stress system affects function within the microbiota-gut-brain axis, and dysregulation of this axis affects function within the stress system. For example, gut microbiota play a role in the programming and activity of the HPA axis_-including gene expression - and influence neuronal activation and the brain stress systems (Butler et al. 2019; Bastiaanssen et al. 2020).

As we see from the above, the microbiota-gut-brain axis is immensely complex; clinicians from non-medical backgrounds may find this chapter challenging. In order to avoid being overwhelmed by the detail, it may be helpful for the reader to shift gear to systems thinking. In this way, the reader can hold in mind, in a general way, that dysregulation of any one of these processes can compromise both healthy gut function and healthy brain function, and can contribute to the generation of functional somatic symptoms. Or, in a nutshell, the reader can think of functional gut disorders as emerging from a 'combination of irritable bowel, [activated communication pathways,] and irritable brain' (Qin et al. 2014, p. 14126; Mayer et al. 2019).

See Online Supplement 1.3 for references to non-technical articles about the microbiota-gut-brain axis published either in the health sections of major newspapers or by other science writers.

\section{What We Know About the Gut: The Gut as a Complex System}

In the late 1990s, the neuroscientist Michael Gershon nicknamed the gut's nervous system the second brain (Gershon 1998). Gershon, who had spent his entire research career studying the gut, worked during an era in which the brain was seen as 'the central organ of stress and 
adaptation' (McEwen 2009, p. 911) - a time when research on the gut was somewhat disparaged. Through his play on words, Gershon wanted to highlight the complexity of the gut system and its important role in human health and disease. The intriguing comparison was also a way of drawing attention, as well as the interest of young scientists, to his field.

The gut has its own nervous system, the enteric nervous system, which includes both sensory and motor components. It is a network of nerve fibres that respond to chemical and mechanical stimuli and that activate smooth muscle in the gastrointestinal tract, pancreas, gall bladder, and blood vessels to coordinate motility, endocrine secretions, and blood flow-together, the gut's digestive and defensive programs. Historically, the enteric nervous system was considered part of the autonomic nervous system, which innervates the viscera: all the organs and tissues inside the body (see Online Supplement 1.2). The gut's motor system is part of the visceromotor system, the efferent component of the autonomic nervous system (see Chapter 6). With the exception of the brain, the gut contains the largest number of neurons in the body.

The gut also functions, in effect, as a barrier between the body and the outside world. In this context, the 'outside world' comprises the microbiota, the colony of organisms that live inside the gut. A large proportion of the body's immune-inflammatory cells reside in the gut lumen, where they help maintain the integrity of the gut barrier. Any loss of gut-barrier integrity can lead to the passage of organisms across the gut wall into the inside of the body (organism translocation due to increased gut permeability), which in turn activates the immune-inflammatory system (resulting in increased inflammation) and also disrupts nutrition, digestion, and absorption (Osadchiy et al. 2019).

Each person's gut microbiota is the community of microorganismsincluding bacteria, fungi, and viruses - that live in that individual's gut at any particular time. The genetic material carried by the microbiota-'the full collection of genes in all the microbes in a microbiota community' (Learn.Genetics, Genetic Science Learning Center 2020)—is called the microbiome. The health and relative stability of the microbiota are important to the health and well-being of the individual because the microbiota lives in symbiosis with humans and works together with the 
human body to maintain life processes. These symbiotic functions are so intertwined that some scientists have suggested that humans (and presumably, therefore, many other animals or at least primates) should be viewed as multi-species organisms and that the microbiome should be considered a second form of genetic inheritance, one that is acquired via the mother's birth canal, through subsequent close body contact during infancy (but also later), from the physical environment in early childhood, from the food that we eat, and from the microorganisms that we encounter in our daily lives, lifelong (Gilbert et al. 2012).

Among other things, a healthy and diverse microbiota helps in the digestion of food, contributes to the gut-barrier integrity by protecting the individual from pathogenic organisms, modulates the immune-inflammatory system and the HPA axis, and supports normal brain development and function (Osadchiy et al. 2019; DominguezBello et al. 2019; Bastiaanssen et al. 2020). For example, lactobacillithe bacteria found in yogurt and fermented milk-help in maintaining a healthy microbiota via their antimicrobial functions (production of antimicrobial molecules), anti-inflammatory functions, and gut-barrier functions (for references see Online Supplement 1.3). Disruption of lactobacillus populations via physical (bad diet or antibiotics) or psychological stress may, in turn, compromise the health and integrity of the gut, and increase its vulnerability to functional somatic symptoms.

Healthy eating contributes to the health and relative stability of the microbiota. In this context, the eating patterns in Western-style societies, with so much processed food and the decreased intake of fruits and vegetables, appears to be decreasing the biodiversity of the human gut microbiota. Some scientists propose that the dwindling health of the microbiota in modern, Western-style societies may, in part, explain their soaring rates of anxiety and depression, as well as the apparent increase in functional gut disorders, other functional somatic symptoms, and other forms of dis-ease (Zinocker and Lindseth 2018). Moderate pleasurable exercise also affects the health of the microbiota and is one of the mechanisms that increase the child's stress tolerance (Bastiaanssen et al. 2020). 


\section{Communication Between the Gut and the Brain}

The microbiota-gut-brain axis, discussed at the outset and also known as the gut-brain axis or brain-gut axis, comprises the many different mechanisms through which the gut and brain communicate with each other. Gut-to-brain communication includes the following: the vagal nerve (the afferent part of the autonomic system) and other interoceptive afferents; messenger molecules secreted by the immune-inflammatory cells (part of the immune-inflammatory system); hormone messengers produced by gut endocrine cells (part of the endocrine system); and other messenger molecules (e.g., neurotransmitters and metabolites) produced by the microbiota or the gut (Foster et al. 2017). Brain-to-gut communication includes the following: the autonomic system (sympathetic, restorative parasympathetic, and defensive parasympathetic) and the HPA axis (see Chapter 6). Via these connections, disruptions of the axis on the gut level will affect brain function, and disruptions of the axis on the brain level will affect gut function.

\section{The Gut and Pain}

Pain is a common symptom in functional gut disorders. The mechanisms that underpin chronic/complex pain in the gut are the same as those that underpin chronic/complex pain elsewhere (see Chapter 9). In chronic or recurring gut pain, the pain system is activated on many levels.

- At the tissue level, pain-sensing nerve endings are kept in an activated state (known as visceral sensitivity, visceral hypersensitivity, or neurogenic inflammation).

- At the spinal cord level, nerve cells that receive and carry information about pain are activated (called central sensitization or neuroinflammation).

- At the brain level, pain maps - central representations of pain-are activated (also called central sensitization or neuroinflammation). 
On all these levels, activation of neurons involved in sensing, carrying, or representing pain is maintained by aberrant activation of the immune-inflammatory system. Hence the idea that an irritable bowel exists alongside an irritable brain (Qin et al. 2014). For further details about chronic/complex pain, see Chapter 9.

\section{Stress-System Activation Affects Healthy Gut Function}

From reading the previous sections, the reader will already have noticed that the stress system and the microbiota-gut-brain axis share many components; they are overlapping systems.

- The autonomic nervous system-made up of the sympathetic system and the restorative parasympathetic and defensive parasympathetic systems - is part of both the stress system and the microbiota-gut-brain axis. Autonomic nerves provide communication from the gut to the brain and from the brain to the gut.

- The immune-inflammatory system is part of both the stress system and the microbiota-gut-brain axis, and a large number of immune cells reside in the gut and communicate to the brain via immune-inflammatory signalling molecules.

- The HPA axis communicates with the gut via hormone messengers. In addition, the gut microbiome affects both the development and the regulation of the HPA axis (Sudo 2014; Bastiaanssen et al. 2020).

Because of this close relationship between the stress system and the microbiota-gut-brain axis, activation or dysregulation of the former will affect the latter-including gut health and function, along with the well-being of the gut microbiota-and will potentially trigger, or contribute to the emergence of, functional gut symptoms (Foster et al. 2017; Osadchiy et al. 2019).

Of particular importance, changes in the activation of the autonomic system significantly affect gut health and well-being. As we saw in Chapter 6, activation of the sympathetic component of the autonomic 
system switches off normal digestive programs in both the upper and lower digestive tract, and activation of the defensive parasympathetic component of the autonomic system activates the defensive programs of nausea, vomiting, and diarrhoea. In addition, activation of the autonomic system during periods of stress may mediate changes that decrease gut-barrier integrity and lead to the loss of healthy microbiota species (Michels et al. 2019). Some of the factors that contribute to the decrease in gut-barrier integrity include the following: reduced secretion of gastric acid, reduced gastric emptying, slower transit in the small intestine, and reduced levels of anti-inflammatory antibodies that specialize in mucosal protection (Campos-Rodriguez et al. 2013). Taken together, the reduction of gut-barrier integrity and its consequences for the microbiota will decrease the health and well-being of the gut, and potentially lead to the emergence of functional gut symptoms. Dysregulation in one component of the microbiota-gut-brain axis can thus have potentially major flow-on effects in other components.

\section{The Microbiota, Health, and Well-Being}

The connection between the health of the microbiota and stress-induced symptoms was identified more than a century ago (for references see Online Supplement 1.3). In 1907, Ilya Metchnikoff, a Ukrainian-Russian biologist and the 1908 Nobel Prize winner in Physiology or Medicine, wrote about the health benefits of yogurt eating - and ingestion of lactobacilli-in rural Bulgaria. And in 1910, the physician George Porter Phillips reported that preparations rich in live lactic acid bacteria-for example, curdled milk or liquid solutions from malted grains-were helping to improve symptoms in his patients with melancholia (severe depression). Lactobacilli, as we saw previously, have multiple antimicrobial, anti-inflammatory, and gut-barrier functions that help in maintaining a healthy microbiota-gut-brain axis. More recently, a small number of randomized, controlled trials suggest that diet-in and of itself-can improve symptoms of depression (Parletta et al. 2019; Opie et al. 2018; Bastiaanssen et al. 2020). Probiotics - made up of live microbes that have a beneficial effect on 
the individual-also appear to have beneficial effects on mood, as reported by Phillips in 1910 (Bastiaanssen et al. 2020). In this context, and to help foster long-term health and well-being, a healthy diet-one that includes a regular top-up of lactobacilli-needs to be a key component in all clinical interventions for functional somatic symptoms.

\section{The Foundation for the Microbiota Is Established Early in Life}

Recent studies show that the health of the gut microbiota is established during the first days, weeks, and months of life. The baby is colonized by the correct type of microbes during the vaginal birth process (including from faeces), and also via microbes picked up from the environment and via breast feeding. The health of the baby's microbiota can be disrupted by maternal use of antibiotics, caesarean delivery, and antibiotic use during early development. The role of microbiota disruptions for subsequent health and disease across the lifespan is a topic of current research. For references see Online Supplement 1.3.

\section{The Microbiota Modulates Set-Points Within the Gut System}

The microbiota plays a centrally important role in the gut by modulating set-points for gut motility, visceral sensitivity, and thickness of the gut's mucosal barrier (Galley et al. 2014). Some of these functions are controlled via serotonin, a neurotransmitter synthesized in the gut and also in the microbiota. Too much serotonin in the gut lumen-because of too much production or inadequate reuptake-can cause abdominal pain and cramping, bloating, and, in some individuals, alternating diarrhoea and constipation (key symptoms of irritable bowel syndrome). Likewise, disruptions in the health of the microbiota presumably affect serotonin production and may contribute to functional presentations. It is notable in this context that many, though not all, studies of individuals with irritable bowel syndrome show unhealthy changes in microbiota composition (Osadchiy et al. 2019; Bastiaanssen et al. 2020). 
In parallel, because serotonin is also a key neurotransmitter in the brain, changes in serotonin metabolism in the gut may affect anxiety, depression, and mood-related behaviours (Faure et al. 2010; O'Mahony et al. 2017). Indeed, some studies of probiotics in patients with high levels of stress, irritable bowel syndrome, and chronic fatigue syndrome have shown, respectively, decreases in stress-related gut symptoms and stress/anxiety measures, better mood scores and decreased responses to fear stimuli, and decreased anxiety symptoms.

For references see Online Supplement 1.3.

\section{The Microbiota Modulates Set-Points Within the Stress System}

The microbiota also modulates set-points within the stress system-for example, the reactivity of the HPA axis (Sudo 2014; Foster et al. 2017; Bastiaanssen et al. 2020). Children with a healthy microbiota may be less susceptible to HPA-axis hyper- or hypo-activation in the face of adversity (see Chapter 8). By keeping stress-system activation within a healthy range, these children may be less susceptive to a broad range of stress-related symptoms and disorders, including anxiety, depression, and functional somatic symptoms of all kinds. For references see Online Supplement 1.3.

\section{Stress and Functional Gut Symptoms}

As we have seen in previous sections, the health and well-being of the gut system relies on the health and well-being of all components that make up the microbiota-gut-brain axis and on communication and synchronization both within and between the microbiota-gut-brain axis and the stress system. In the vignettes below we provide some clinical examples so that the reader can see how these issues present in clinical practice.

In clinical practice, functional gut symptoms-like other functional symptoms - are often triggered by psychological stress. The vignette of 
Tommie illustrates how psychological stress activated Tommie's stress system, which then activated his gut and led to his presentation with pain (headache) and gut symptoms.

Tommie was an eight-year-old boy in primary school. He was a good student who liked to please his teachers. Tommie told the following story. In year 2 of primary school, he had had a 'nice' teacher and a good year at school (resulting in a stress rating of $1 / 10$ for Tommie). In year 3 his teacher changed, and Tommie and the class did not like her (stress rating of 9/10 for Tommie). Tommie said that she disapproved of how the class dressed, and frequently made the children stay back at lunch. Among the many things she disliked were the bags that the children used to carry their things, the way that they placed their bags in the classroom, and the types of pens that they used. Tommie found himself unable to please his teacher. He began to worry about going to school, and he also began to suffer from headaches and nausea. He had butterflies and cramps in his stomach every day, and sometimes he also vomited. One day, the teacher lost her temper, and she expressed her disapproval of Tommie publicly in front of the class. That evening Tommie's mother brought him to the emergency department clutching his head in pain. When Tommie told the story, his body manifest its distress. Tommie first showed increases in both respiratory and heart rates. Then the headache increased in intensity, and Tommie clutched his head in distress. Then Tommie's abdomen made fluttering motions that were visible to the interviewer (presumably reflecting movement of smooth muscle that makes up the gut). A little later, Tommy retched into a vomit bag. Finally, Tommie's arms and legs went weak. His mother gathered him into her arms, and Tommie sobbed into her body.

In clinical practice, functional gut symptoms and the dysregulation of the microbiota-gut-brain axis can also be triggered by physical stress such as an illness or infection, as we saw in the vignette of Martin in Chapter 9. In this way, even though the infection or illness resolves, ongoing symptoms may be maintained by ongoing dysregulation of the microbiota-gut-brain axis or ongoing activation or dysregulation of the stress system (or components of the stress system). Ongoing dysregulation of the microbiota-gut-brain axis makes the child more susceptive to subsequent episodes of infection and to symptoms of anxiety and depression. 
As we see in the following vignette of Emma, some patients walk a difficult path and demonstrate remarkable tenacity and resilience in the face of a series of physical insults involving the gut that have eventually triggered functional somatic symptoms. While the medical details of the presentation may be challenging to the reader, it is important to include them here because Emma's pattern of presentation is one that gastroenterologists encounter on a regular basis.

Emma was an 18-year-old woman with a five-year history of chronic abdominal pain and recurring episodes of nausea, bringing up food (sometimes via vomiting and sometimes via rumination [see Chapter 7]), and irregular bowel function. Emma's initial period of illness followed in the wake of various medical events: an episode of spontaneous peritonitis (inflammation of the inner lining of the bowel) diagnosed via laparoscopy (keyhole surgery using a camera), which was followed by multiple treatments with various antibiotics. The symptoms of severe vomiting and explosive diarrhoea — and the inability to keep food down-had worsened after a gastric-emptying study (study looking at mobility of the stomach) that used a radioactive preparation to which Emma had a serious adverse response. Investigations suggested that gut dysmotility (loss of normal speed and rhythm of bowel movements) and visceral hypersensitivity (activation of the pain system on the gut system level) contributed to the clinical picture. Emma also suffered from severe postural orthostatic tachycardia syndrome (POTS), a form of autonomic dysregulation that made it more difficult for her to mobilize from her bed (see Chapter 6). In the early stages of the illness, her respiratory motor system had been chronically activated (chronic hyperventilation with a slightly low $\mathrm{pCO}_{2}$ [see Chapter 7]). Comorbid anxiety contributed to her difficulties with both falling asleep and remaining asleep at night. New episodes of illness were triggered by infections such as colds and bouts of gastritis. But Emma was keen to be well, and she had engaged actively in treatment, availing herself of all the suggested interventions for treating her symptoms: pharmacological (e.g., medications for gut function, POTS, and anxiety), physical (e.g., physiotherapy and nasogastric tube to top up her hydration status), and psychological (e.g., mind-body pain-regulation strategies). After each new bout of illness, Emma had to work extra hard to regain her previous level of function and to maintain periods of well-being. In the future, new options, such as faecal transplants and a targeted use of probiotics, may be added to treatment plans to help patients like Emma. 
Throughout the book we have emphasized the interrelated nature of functional somatic symptoms and how different symptoms can exist one alongside the other. An additional feature of functional somatic symptoms is their propensity to change over time. In the following vignette of Morgan, we see how the somatic symptoms-including symptoms involving the microbiota-gut-brain axis-changed over time with exposure to new stress. The vignette highlights that failure to treat functional somatic symptoms - and to address issues in the child's family and social context-may result in a complex clinical picture in which symptoms layer over symptoms, leaving the child progressively more disabled. The vignette also highlights in a tangible way the connection between ACEs and increased risk for functional somatic symptoms in both children and adults (for a discussion of ACEs, see Chapter 4).

Morgan, a 15-year-old girl living with her parents, had an eight-year history of functional gut symptoms: recurrent abdominal pain, nausea, and vomiting; ongoing difficulties with eating; and irregular bowel function. During the family assessment, Morgan and her family gave a history of chronic stress at home because her parents were always fighting. At the age of 12, Morgan experienced a sporting injury - a fall that involved a severe knock to the head-and her symptom pattern became more complicated. She developed chronic headache and fatigue, and began to suffer from intermittent non-epileptic seizures. Since she found the treatment interventions offered by her local hospital (involving a pain team and mental health team) unhelpful, she stopped attending appointments. At the age of 15 , after a period of nasty bullying at school, she presented to hospital with functional hemiparesis and sensory loss of the left side of her face and body.

In this chapter we have seen that the human body is not all our own. We share this body with microscopic organisms that live on every crevice and contour of our body: on our skin, on all our mucous membranes, and, most especially, inside our gut. We have seen how gut doctors have had to shift their thinking from the gut as a single organ to the gut as part of a complex system, the microbiota-gut-brain axis. We have also seen that functional gut disorders-expressed in symptoms 
of nausea, vomiting, abdominal pain, cramping, bloating, irregular bowel function, alternating diarrhoea and constipation, and so onare now conceptualized as disorders arising from dysregulation of the microbiota-gut-brain axis. Both physical and psychological stress can function as triggers that dysregulate the microbiota-gut-brain axis away from physiological coherence and health to a state of imbalance and disharmony that may be expressed in functional gut symptoms.

\section{References}

Bastiaanssen, T. F. S., Cussotto, S., Claesson, M. J., Clarke, G., Dinan, T. G., \& Cryan, J. F. (2020). Gutted! Unraveling the Role of the Microbiome in Major Depressive Disorder. Harvard Review of Psychiatry, 28, 26-39.

Bradford, K., Shih, W., Videlock, E. J., Presson, A. P., Naliboff, B. D., Mayer, E. A., \& Chang, L. (2012). Association Between Early Adverse Life Events and Irritable Bowel Syndrome. Clinical Gastroenterology and Hepatology, 10, 385-390.e3.

Butler, M. I., Cryan, J. F., \& Dinan, T. G. (2019). Man and the Microbiome: A New Theory of Everything? Annual Review of Clinical Psychology, 15, 371-398.

Campos-Rodriguez, R., Godinez-Victoria, M., Abarca-Rojano, E., Pacheco-Yepez, J., Reyna-Garfias, H., Barbosa-Cabrera, R. E., \& Drago-Serrano, M. E. (2013). Stress Modulates Intestinal Secretory Immunoglobulin A. Frontiers in Integrative Neuroscience, 7, 86.

Dominguez-Bello, M. G., Godoy-Vitorino, F., Knight, R., \& Blaser, M. J. (2019). Role of the Microbiome in Human Development. Gut, 68, 1108-1114.

Drossman, D. A. (2016). Functional Gastrointestinal Disorders: History, Pathophysiology, Clinical Features and Rome IV. Gastroenterology, 150, 1262-1279.e2.

Drossman, D. A., \& Hasler, W. L. (2016). Rome IV-Functional GI Disorders: Disorders of Gut-Brain Interaction. Gastroenterology, 150, 1257-1261.

Faure, C., Patey, N., Gauthier, C., Brooks, E. M., \& Mawe, G. M. (2010). Serotonin Signaling Is Altered in Irritable Bowel Syndrome with Diarrhea but Not in Functional Dyspepsia in Pediatric Age Patients. Gastroenterology, 139, 249-258.

Foster, J. A., Rinaman, L., \& Cryan, J. F. (2017). Stress \& the Gut-Brain Axis: Regulation by the Microbiome. Neurobiology of Stress, 7, 124-136. 
Galley, J. D., Nelson, M. C., Yu, Z., Dowd, S. E., Walter, J., Kumar, P. S., et al. (2014). Exposure to a Social Stressor Disrupts the Community Structure of the Colonic Mucosa-Associated Microbiota. BMC Microbiology, 14, 189.

Gershon, M. (1998). The Second Brain: The Scientific Basis of Gut Instinct and a Groundbreaking New Understanding of Nervous Disorders of the Stomach and Intestine. New York: HarperCollins.

Gilbert, S. F., Sapp, J., \& Tauber, A. I. (2012). A Symbiotic View of Life: We Have Never Been Individuals. Quarterly Review of Biology, 87, 325-341.

Hyams, J. S., Di Lorenzo, C., Saps, M., Shulman, R. J., Staiano, A., \& Van Tilburg, M. (2016). Childhood Functional Gastrointestinal Disorders: Child/Adolescent. Gastroenterology, 150, 1456-1468.e2.

Learn.Genetics, Genetic Science Learning Center. (2020). The Human Microbiome. https://learn.genetics.utah.edu/Content/Microbiome/.

Mayer, E. A., Labus, J., Aziz, Q., Tracey, I., Kilpatrick, L., Elsenbruch, S., et al. (2019). Role of Brain Imaging in Disorders of Brain-Gut Interaction: A Rome Working Team Report. Gut, 68, 1701-1715.

McEwen, B. S. (2009). The Brain Is the Central Organ of Stress and Adaptation. Neuroimage, 47, 911-913.

Michels, N., Van De Wiele, T., Fouhy, F., O’Mahony, S., Clarke, G., \& Keane, J. (2019). Gut Microbiome Patterns Depending on Children's Psychosocial Stress: Reports Versus Biomarkers. Brain, Behavior, and Immunity, 80, 751-762.

O’Mahony, S. M., Dinan, T. G., \& Cryan, J. F. (2017). The Gut Microbiota as a Key Regulator of Visceral Pain. Pain, 158(Suppl 1), S19-S28.

Opie, R. S., O’Neil, A., Jacka, F. N., Pizzinga, J., \& Itsiopoulos, C. (2018). A Modified Mediterranean Dietary Intervention for Adults with Major Depression: Dietary Protocol and Feasibility Data from the Smiles Trial. Nutritional Neuroscience, 21, 487-501.

Osadchiy, V., Martin, C. R., \& Mayer, E. A. (2019). The Gut-Brain Axis and the Microbiome: Mechanisms and Clinical Implications. Clinical Gastroenterology and Hepatology, 17, 322-332.

Park, S. H., Videlock, E. J., Shih, W., Presson, A. P., Mayer, E. A., \& Chang, L. (2016). Adverse Childhood Experiences Are Associated with Irritable Bowel Syndrome and Gastrointestinal Symptom Severity. Neurogastroenterology and Motility, 28, 1252-1260.

Parletta, N., Zarnowiecki, D., Cho, J., Wilson, A., Bogomolova, S., Villani, A., et al. (2019). A Mediterranean-Style Dietary Intervention Supplemented with Fish Oil Improves Diet Quality and Mental Health in People with Depression: A Randomized Controlled Trial (Helfimed). Nutritional Neuroscience, 22, 474-487. 
Qin, H. Y., Cheng, C. W., Tang, X. D., \& Bian, Z. X. (2014). Impact of Psychological Stress on Irritable Bowel Syndrome. World Journal of Gastroenterology, 20, 14126-14131.

Sudo, N. (2014). Microbiome, HPA Axis and Production of Endocrine Hormones in the Gut. Advances in Experimental Medicine and Biology, 817, 177-194.

Varni, J. W., Lane, M. M., Burwinkle, T. M., Fontaine, E. N., Youssef, N. N., Schwimmer, J. B., et al. (2006). Health-Related Quality of Life in Pediatric Patients with Irritable Bowel Syndrome: A Comparative Analysis. Journal of Developmental and Behavioral Pediatrics, 27, 451-458.

Zinocker, M. K., \& Lindseth, I. A. (2018). The Western Diet-MicrobiomeHost Interaction and Its Role in Metabolic Disease. Nutrients, 10, 365.

Open Access This chapter is licensed under the terms of the Creative Commons Attribution-NonCommercial-NoDerivatives 4.0 International License (http://creativecommons.org/licenses/by-nc-nd/4.0/), which permits any noncommercial use, sharing, distribution and reproduction in any medium or format, as long as you give appropriate credit to the original author(s) and the source, provide a link to the Creative Commons license and indicate if you modified the licensed material. You do not have permission under this license to share adapted material derived from this chapter or parts of it.

The images or other third party material in this chapter are included in the chapter's Creative Commons license, unless indicated otherwise in a credit line to the material. If material is not included in the chapter's Creative Commons license and your intended use is not permitted by statutory regulation or exceeds the permitted use, you will need to obtain permission directly from the copyright holder.

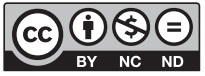

